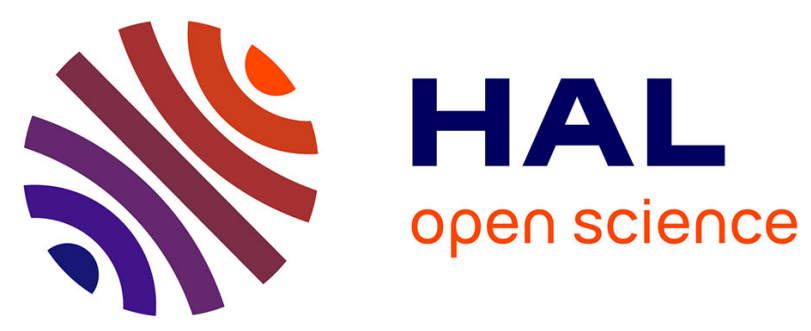

\title{
A fair cluster-based resource and power allocation scheme for two-tier LTE femtocell networks
} Amira Bezzina, Mouna Ayari, Rami Langar, Leila Azouz Saidane

\section{To cite this version:}

Amira Bezzina, Mouna Ayari, Rami Langar, Leila Azouz Saidane. A fair cluster-based resource and power allocation scheme for two-tier LTE femtocell networks. Global Information Infrastructure and Networking Symposium (GIIS) 2016, Oct 2016, Porto, Portugal. 10.1109/GIIS.2016.7814945 . hal01529345

\section{HAL Id: hal-01529345 \\ https://hal.sorbonne-universite.fr/hal-01529345}

Submitted on 30 May 2017

HAL is a multi-disciplinary open access archive for the deposit and dissemination of scientific research documents, whether they are published or not. The documents may come from teaching and research institutions in France or abroad, or from public or private research centers.
L'archive ouverte pluridisciplinaire HAL, est destinée au dépôt et à la diffusion de documents scientifiques de niveau recherche, publiés ou non, émanant des établissements d'enseignement et de recherche français ou étrangers, des laboratoires publics ou privés. 


\title{
A Fair Cluster-based Resource and Power Allocation Scheme for Two-tier LTE Femtocell Networks
}

\author{
Amira BEZZINA $^{1,2}$, Mouna AYARI $^{1,2}$, Rami LANGAR ${ }^{3}$, and Leila AZOUZ SAIDANE ${ }^{2}$ \\ ${ }^{1}$ Sorbonne Universities, UPMC Univ Paris 06, CNRS, LIP6 UMR 7606, 4 place Jussieu 75005 Paris, France. \\ ${ }^{2}$ CRISTAL Lab., National School of Computer Sciences, Univ. of Manouba,Tunisia. \\ ${ }^{3}$ LIGM/UPEM - University Paris Est; Cité Descartes, 77454 Marne-la-Vallée, France. \\ Emails: \{amira.bezzina, mouna.ayari\}@lip6.fr, rami.langar@u-pem.fr, leila.saidane@ensi.rnu.tn
}

\begin{abstract}
In this paper, we propose a joint power control and resource allocation approach for co-channel deployed femto- and macro-cell networks, denoted as Two-tier Clusterbased Resource and Power Allocation Scheme (TCRPA). The rational behind our proposal is to efficiently mitigate the significant interference faced by indoor users in downlink communications in order to satisfy the resource allocation requirements of femto-users (FU). To this end, we first formulate the resulting optimization problem as a MixedInteger Linear Program (MILP). Then, we provide a semicentralized scheme to solve it. Our proposed scheme TCRPA is based on clustering, which is an arrangement aiming to take the advantages of both centralized and distributed approaches. Simulation results demonstrate that TCRPA can efficiently allocate power and fairly distribute spectrum resources to FUs without affecting the quality of the active macro-users (MU) communications. In particular, significant gains in terms of the overall throughput and the SINR level are achieved in comparison with two prominent state of the art solutions.
\end{abstract}

Index Terms-Femtocell, OFDMA, downlink, two-tier, interference mitigation, resource allocation, power control

\section{INTRODUCTION}

Due to the growth of the indoor users' requests for high speed and high data-rate services such as video on demands and VoIP, femtocell deployment emerged as a promising solution to arise the network capacity and improve its coverage with a low financial cost for operators. These home base stations, commonly known as femtocells, are an attractive low financial cost option for mobile operators to address the mentioned issues by simply making the transmitter and the receiver closer to each other. However, since femtocells are already widely installed in households and offices today, their coexistence and the short proximity of their users may undoubtedly result in an important co-tier interference. Besides, the installation of femtocells in the underlaying macrocell network gives birth to a cross-tier interference when femtocells and macrocells use the same spectrum. The latter issue can be treated by using different fre- quency bands for femto- and macro-cells. But, splitting the spectrum may lower the reuse of the available resources and the spectral efficiency. Hence, managing the two types of interference in a co-channel environment is a heavy task that operators must face especially in dense urban networks. In this type of scenario, congestion can occur when femto-users' demands exceed the available resources. As around $80 \%$ of all mobile broadband traffic is consumed by users located indoors [1] and a femtocell can serve upon 8 to 10 users, congestion can probably take place. Thus, an adapted resource and power allocation scheme especially for indoor users (i.e femto-users) is mandatory to stand up to the interference they may face.

In this paper, we tackle this important issue and we propose a new two-tier cluster-based resource and power allocation (RPA) approach denoted as TCRPA. We first formulate TCRPA as a Mixed-Integer Linear Program (MILP) that considers a trade-off between the following costs: (i) minimizing the transmit power of femtocells, and (ii) maximizing the resource allocation regarding to an SINR threshod. Then, we propose a cluster-based approach to solve it. The effectiveness of our solution is then demonstrated using a quite congested urban topology. As main observations, TCRPA retains good performance with respect to macro-users. On the other hand, improvements in the overall femtousers' throughput and SINR are noticed in addition to the decrease of the transmit power of FAPs and a fair resource distribution between the tiles.

The remainder of the paper is organized as follows. Section II discusses literature that is relevant to this work. Section III presents the system model used in our approach and the problem formulation as an optimization problem. The MILP proposed algorithm is then introduced. Simulation results are provided in section IV. Finally, section V concludes this paper. 


\section{RELATED WORK}

In the few last years, extensive research efforts have been dedicated towards finding efficient resource allocation scheme to avoid as much as possible the femtocell interference in Orthogonal Frequency Division Multiple Access (OFDMA)-based networks for downlink communications [2], [3].

Authors of [4] have opted for using a partially shared spectrum to mitigate the cross-tier interference. In [5], a novel RPA that uses resource isolation in the frequency domainwas presented. As aforementioned, this kind of schemes may result in low spectral efficiency. Thus, cochannel femtocell deployments are preferred for making an efficient spectral reuse and providing more flexibility in a dense environment. Regardless the spectrum use, three types of strategies have been studied: distributed, centralized and semi-centralized. Studying especially dense networks, the latter approach seems to be an interesting solution for reducing the co-tier interference by coordinating the transmissions of FAPs by slicing the problem into a set of simple sub-problems.

Many clustering methods proposed for interference mitigation schemes have been proposed in the literature. [6] proposed a joint power control and resource allocation algorithm based on clusters named QP-FCRA taking into consideration FUs' QoS requirements. In [7], authors proposed a promising low complexity clusterbased algorithm using semi-definite programming to manage the co-tier interference. In [8], authors proposed a dynamic clustering-based sub-band allocation scheme for dense femtocell environment. However, they ignored the power and focused on frequency allocation. [9] addresses the interference problem in dense networks by proposing a cluster-based resource allocation problem that maximizes the sum of the femtocell users' throughput under the consideration of co-tier interference mitigation. Moreover, all the later studies ignore cross-tier interference and then implicitly consider the split-spectrum strategy.

\section{INTERFERENCE MANAGEMENT IN TWO-TIER FEMTOCELL NETWORKS}

Although several solutions have already been proposed in the literature, the RPA problem is still an active research field due to the lack of a generic solution which can perform greatly in all urban settings. In this context, we propose in this research work a novel two-tier cluster-based RPA solution called TCRPA. The novelty of our contribution lies on balancing both the power and the resource optimization in the same objective function of the formulated MILP, and the way we easily handle the cross-tier interference.

\section{A. Context and problem formulation}

In this study, we consider a dense OFDMA (e.g., LTE) femtocell network consisting of a macro base station
(MBS) and several access femtocells (FAPs) representing residential or enterprise networks. In such system, the frame structure relies on time-frequency resources blocks (also called RBs or tiles). This work gives an interesting extension to the work in [6] by focusing on both crosstier and co-tier interference mitigation for FUs for downlink communications. Each cell (FAP or Macro) serves a number of users. FU demands represent the required bandwidth expressed in number of required tiles. We assume that MUs have already been served by their MBS. In the following, we first present the notations used in our model. Then, we expose the corresponding SINR formulation and the Optimization Problem.

1) Notations:

- $M$ is the MBS deployed in the network.

- $F=\left\{F_{1}, \ldots, F_{N}\right\}$ is the set of FAPs, where $\mathrm{N}$ is the total number of femtocells deployed in the network.

- $I_{n}$ denotes the interference set of $F_{n} \in F$, which corresponds to the set composed of $F_{n}$ and the femtocells and macrocells causing interference to users attached to $F_{n}$. We note that interference is not symmetric since it depends on user positions.

- $U_{M}$ is the set of users attached to the MBS $M$.

- $U_{n}$ is the set of users attached to the FAP $F_{n}$.

- $d_{u}^{n}$ denotes the demand of femto-user $u \in U_{n}$.

- $D_{n}=\sum d_{u}^{n}$ denotes the demand of the FAP $F_{n}$.

- $K=\left\{K_{1}, \ldots, K_{N}\right\}$ is the set of available tiles.

- $\Delta_{n, u}^{k}$ is the binary resource allocation variable for femtouser $u \in U_{n}$ which is set to 1 if the tile $k$ is used, and 0 otherwise.

- $P_{n, u}^{k}$ is the transmit power allocated from FAP $F_{n}$ to its user $u$ on tile $k$, where $P_{n, u}^{k} \geq P_{\min }^{F}$ if the tile $k$ is used by user $u$, or $P_{n, u}^{k}=0$ otherwise.

- $P_{M, u}^{k}$ is the transmit power allocated from the MBS $M$ to its user $u$ on tile $k$, where $P_{M, u}^{k} \geq P_{\min }^{M}$ if the tile $k$ is used by user $u$, or $P_{M, u}^{k}=0$ otherwise.

- $P_{m i n}^{F}$ is the minimum required transmit power per tile for a successful transmission of a FAP.

- $P_{\text {max }}^{F}$ is the total power constraint per femtocell.

- $p_{n, u}^{k}$ is the received power at the FU $u \in U_{n}$ on tile $k$.

- $p_{M, u}^{k}$ is the received power at the MU $u \in U_{M}$ on tile $k$.

- $\Gamma_{u, k}$ is the required SINR for user $u$ on tile $k$.

- $\sigma^{2}$ is the noise density.

- $p l(x, y)$ is the path loss between $x$ and $y$.

- $G_{x}$ is the antenna gain of $x$.

2) SINR formulation: We take into consideration the worst case scenario where the access to the FAPs is closed. In a general context, we can express the SINR of the FU $u \in U_{n}$ attached to the FAP $F_{n}$ as follows:

$\forall k, \forall F_{n} \in F, \forall u \in U_{n}, S I N R_{u, k}=\frac{p_{n, u}^{k}}{I_{M F}+I_{F F}+\sigma^{2}} \geq \Gamma_{u, k}$

where $I_{M F}$ represents the interference due to the transmission of the MBS $M$ to the neighboring MU $v$ on the tile $k$ and $I_{F F}$ represents the interference imposed by the transmission of the neighboring FAPs on the tile $k$, and are calculated as follows:

$$
p_{n, u}^{k}=\frac{P_{n, u}^{k} \times G_{F_{n}} \times G_{u}}{p l\left(F_{n}, u\right)}
$$




$$
\begin{gathered}
\forall v \in U_{M}, I_{M F}=\frac{P_{M, v}^{k} \times G_{M} \times G_{u}}{p l(M, u)} \\
\forall F_{n^{\prime}} \in F, \forall u^{\prime} \in U_{n^{\prime}}, I_{F F}=\sum_{n^{\prime} \neq n} \frac{P_{n^{\prime}, u^{\prime}}^{k} \times G_{F_{n^{\prime}}} \times G_{u}}{p l\left(F_{n^{\prime}}, u\right)}
\end{gathered}
$$

For a MU $u \in U_{M}$, the SINR is expressed as follows:

$$
\forall k, \forall v \in U_{M}, S I N R_{v, k}=\frac{p_{M, v}^{k}}{I_{F M}+\sigma^{2}} \geq \Gamma_{v, k}
$$

where $I_{F M}$ represents the interference due to the transmission of the neighboring FAPs using the same tile $k$.

$$
\begin{gathered}
p_{M, v}^{k}=\frac{P_{M, v}^{k} \times G_{M} \times G_{v}}{p l(M, v)} \\
\forall F_{n} \in F, \forall u \in U_{n}, I_{F M}=\sum_{F_{n} \in F} \frac{P_{n, u}^{k} \times G_{F_{n}} \times G_{v}}{p l\left(F_{n}, v\right)}
\end{gathered}
$$

3) Related Optimization Problem: As already mentioned, in urban dense environment, we expect that the overall demand of cells is often higher than the available resources. Therefore, our objective is to find, for such congestion situations, a strategic resource and power allocation scheme that satisfies throughput expectations while controlling the interference between MUs and FUs (cross-tier interference) as well as between FUs themselves (co-tier interference). The corresponding optimization problem is as follows.

$$
\text { Minimize } \sum_{u \in U_{n}} \sum_{k}\left(\alpha \times \frac{P_{n, u}^{k}}{P_{m a x}^{F}}-(1-\alpha) \Delta_{n, u}^{k}\right)
$$

\section{Subject to:}

$$
\begin{aligned}
& \text { (a) } \forall k, \forall F_{n} \in F, \forall u \in U_{n}, \forall u^{\prime} \in U_{n^{\prime}}, \forall v \in U_{M}: \\
& P_{n, u}^{k} \geq \frac{\Gamma_{u, k} \times p l\left(F_{n}, u\right)}{G_{F_{n}} \times G_{u}} \times\left(\frac{P_{M, v}^{k} \times G_{M} \times G_{u}}{p l(M, u)}\right. \\
& \left.+\sum_{n^{\prime} \neq n} \frac{P_{n^{\prime}, u^{\prime}} \times G_{F_{n^{\prime}}} \times G_{u}}{p l\left(F_{n^{\prime}}, u\right)}+\sigma^{2}\right)-\left(1-\Delta_{n, u}^{k}\right) \times R \times P_{\max }^{F} \\
& \text { (b) } \forall k, \forall F_{n} \in F, \forall u, v \in U_{n}: \Delta_{n, u}^{k}+\Delta_{n, v}^{k} \leq 1 \\
& \text { (c) } \forall F_{n} \in F, \forall u, \in U_{n}: \sum_{k} \Delta_{n, u}^{k} \leq d_{u}^{n} \\
& \text { (d) } \forall F_{n} \in F: \sum_{u \in U_{n}} \sum_{k} P_{n, u}^{k} \leq P_{\text {max }}^{F} \\
& \text { (e) } \forall k, \forall F_{n} \in F, \forall u \in U_{n}: P_{n, u}^{k} \geq \Delta_{n, u}^{k} \times P_{\min }^{F} \\
& \text { (f) } \forall k, \forall F_{n} \in F, \forall u \in U_{n}: \Delta_{n, u}^{k} \in\{0,1\}
\end{aligned}
$$

Condition (a) denotes that the transmit power on tile $\mathrm{k}$ should guarantee the required SINR. The second term on the right hand of the inequality (a) ensures that the transmit power is equal to 0 if $\Delta_{n, u}^{k}=0$, where $\mathrm{R}$ is a carefully chosen very high value. If the tile is in use then the second parts of the inequalities turn to zero and the transmit power gets the required value. Condition (b) ensures that two users attached to the same unit (i.e FAP) cannot use the same tile. Condition (c) indicates that a user cannot obtain more than what he demands.
Conditions (d) and (e) refer to the power constraints, and finally condition (f) indicates that $\Delta_{n, u}^{k}$ is a binary variable.

The problem is formulated as a MILP since the objective function is a sum of two weighted outputs. To solve it, we propose to subdivide it into sub-problems by means of clustering. The corresponding problem will be then solved sequentially. By using such a process, we considerably reduce the time complexity of the allocation problem. In the following, we detail all the steps of our TCRPA related algorithm.

4) TCRPA with K-Means Clustering: TCRPA algorithm consists of two main phases: the cluster formation and the intra-cluster resource and power allocation.

Based on the femtocells positions, the network will be partitioned. Femtocells are divided into disjoint clusters, where the entire tiles are available for each cluster. Note that, in the same cluster, two femtocells can not transmit on the same RBs. As clustering algorithm, we use the Kmeans algorithm introduced in [10], which executes an iterative, data-partitioning algorithm based on a given cluster size and cluster number. This algorithm has a complexity of $\mathrm{O}(\mathrm{n})$, with $\mathrm{n}$ is the number of all FUs.

Once the femtocell network is partitioned into clusters, the second step is to jointly allocate resources and transmit power for all FAPs within each cluster taking into account QoS requirements and cross-tier interference state of attached users. To achieve this, each FAP will collect information of the surrounding environment by sniffing the neighboring transmissions and gathering measurement reports received from its attached users. In this step, the maximum transmit power $P_{\max }$ is assumed to be used by all FAPs. Then, each cluster member reports to its corresponding centroid (i.e. clusterhead) the required resources to satisfy its users' demands. Based on these information, the FAP will know the number of its interfering femtocells and macro-users and can then transmit them to the dedicated node. Finally, the joint power control and resource allocation problem is individually resolved by each centroid every $t$ time, which depends on the arrival/departure process of end users residing in the cluster.

\section{Performance Evaluation}

In this section, we evaluate the efficiency of our proposed Two-tier Cluster-based Resource and Power Assignment approach TCRPA via extensive simulation experiments on a highly congested scenario. To prove the forcefulness of our approach, we compare its performance to the distributed resource and power allocation scheme DRAPM [11] with a contention phase and the semi-centralized QP-FCRA [6] approach previously presented. It is worth noting that the modulation and coding scheme adaptation of DRAPM was not taken into consideration to allow a fair comparison between all schemes. Also, the resource contention avoidance 
was not considered for both QP-FCRA and TCRPA. The reported results are obtained using Matlab Tools and AMPL/CPLEX as solver. Simulations were run 100 times.

In the following, we first present the simulation environment and metrics. Then, we discuss the simulation results.

\section{A. Simulation settings}

In order to evaluate the performance of TCRPA, we consider in our experiments a dense network size of 100 FAPs distributed in a $100 \mathrm{~m} \times 200 \mathrm{~m}$ area with a unique Macro Base Station in the middle of the field. This quite congested urban scenario has $50 \%$ indoor users (FUs) and 50\% outdoor users (MUs). In this specific topology, offices/houses and streets are alternated. Each FAP occurs in the middle of the $10 \times 10 \mathrm{~m}^{2}$ residential cell and can serve 8 FUs randomly placed within the cell. Macro-users are randomly placed in the streets.

Femto-users demands $d_{u}^{n}$ depend on the application type they want to use. Each type of application has a specific need in terms of RBs. The table I enumerates the different types of applications and their required bandwidth and RBs. We took into consideration the traffic types and their approximate percentages based on the study [12].

\begin{tabular}{|l|l|l|l|}
\hline $\begin{array}{l}\text { Application } \\
\text { Category }\end{array}$ & $\begin{array}{l}\text { Maximum Required } \\
\text { bandwidth (Mbps) }\end{array}$ & Traffic \% & Number of RBs \\
\hline Data/VoIP/Web & 1 & $36 \%$ & 2 \\
\hline Video & 4 & $55 \%$ & 5 \\
\hline File Sharing & 0.5 & $1 \%$ & 1 \\
\hline Audio Sharing & 0.4 & $8 \%$ & 1 \\
\hline
\end{tabular}

TABLE I

APPLICATION TYPES, PERCENTAGES AND REQUIRED RESOURCES

This analysis was achieved using a downlink LTE frame with a channel bandwidth of $10 \mathrm{Mhz}$ consisting of 50 tiles. The Number of required tiles is computed as follows: $d_{n}^{u}=\frac{T P_{u}^{r e q}}{\psi \times e f f_{u}}$

with:

- $T P_{u}^{r e q}$ is the required bandwidth. This parameter depends on the type of application of the user equipment.

- $\psi=\left(S C_{o f d m} \times S Y_{\text {ofdm }} / T_{\text {subframe }}\right)$ is a fixed parameter that depends on the network configuration.

Having the following values: $S C_{o f d m}=12, S Y_{o f d m}=$ $7, T_{\text {subframe }}=0.5 \mathrm{~ms}$ and ef $f_{u}=4.5 \mathrm{bits} / \mathrm{symbol}$ (the efficiency when using a 64 QAM modulation with a coding rate of $3 / 4$ (MCS13)), the required bandwidth per RB is equal to 756 Kbps. The SINR threshold taken in our MILP is $20 d B$ and the pathloss level is computed using the ITU-R pathloss model [13]. More simulation parameters are reported in the table II.

\begin{tabular}{|l|l|}
\hline Parameter & Value \\
\hline Number of FUs & 800 \\
\hline Number of MUs & 800 \\
\hline Thermal Noise $\sigma^{2}$ & $-121.45 d B m$ \\
\hline Carrier frequency & $2 G h z$ \\
\hline$P_{\max }^{F}$ & $20 m W$ \\
\hline$P_{\min }^{F}$ & $0.1 m W$ \\
\hline$P_{m a x}^{M a c r o}$ & $40 W$ \\
\hline MBS antenna Gain $G_{M B S}$ & $18 d B i$ \\
\hline$G_{F A P}, G_{F U}, G_{M U}$ & $0 d b i$ \\
\hline$\alpha$ & 0.5 \\
\hline
\end{tabular}

TABLE II

SIMULATION PARAMETERS

\section{B. Performance Metrics}

To fully assess the benefits of our proposal, different relevant performance metrics have been chosen:

- The SINR level: It refers to the final SINR level of FUs and MUs computed using the equations 1 and 5 respectively.

- The throughput: To estimate the throughput, we compute the transmission rate to a user $i$ given by the Shannon formula for a Gaussian channel as follows: $C_{i}=W\left(1+\alpha S I N R_{i}\right)$ where $W$ is the available bandwidth for user $i$ and $\alpha$ is a constant for a target bit error rate (BER) defined by $\alpha=\frac{-1.5}{\ln (5 B E R)}$. Here we set BER to $10^{-6}$.

- The transmit power: The level of transmission power of a FAP.

- The Spectrum Spatial Reuse (SSR): It denotes the number of possible FUs using simultaneously the same elementary tile within the network.

\section{Performance Results}

In this section we describe the results of our experiments regarding the metrics described above and we study the important impact of cross-tier interference on resource allocation by comparing our cluster-based proposal to two important approaches.

1) SINR analysis: Studying the final users' SINR values enables us to explore the real impact of the RPA approaches on users' signal level while considering both co- and cross-tier interference.

\begin{tabular}{|l|l|l|}
\hline & Mean FU SINR (dB) & Mean MU SINR (dB) \\
\hline TCRPA & $29.4838 \pm 0.0103$ & $59.0744 \pm 0.0234$ \\
\hline QP-FCRA & $-96.2686 \pm 0.4144$ & $60.0683 \pm 0.0182$ \\
\hline DRAPM & $-94.0008 \pm 0.38$ & $58.0420 \pm 0.0079$ \\
\hline
\end{tabular}

TABLE III

SINR MEAN VALUES

\begin{tabular}{|l|l|l|l|l|l|}
\hline & $\begin{array}{l}\text { Not } \\
\text { connected }\end{array}$ & SINR $\leq 0$ & $\begin{array}{l}0<\text { SINR } \\
\leq 13 \mathrm{~dB}\end{array}$ & $\begin{array}{l}13<\text { SINR } \\
\leq 20 \mathrm{~dB}\end{array}$ & $\begin{array}{l}\text { SINR } \\
>20 \mathrm{~dB}\end{array}$ \\
\hline TCRPA & $70.03 \%$ & $0.15 \%$ & $0.4 \%$ & $0.05 \%$ & $29.37 \%$ \\
\hline QP-FCRA & $69.43 \%$ & $27.77 \%$ & $1.4 \%$ & $0.1 \%$ & $1.3 \%$ \\
\hline DRAPM & $67.51 \%$ & $29.44 \%$ & $1.48 \%$ & $0.22 \%$ & $1.35 \%$ \\
\hline
\end{tabular}

TABLE IV

SINR STUDY 
Tables III and IV clearly show the gain achieved by TCRPA in comparison with solutions that ignore the cross-tier interference. Using TCRAP, the resulting SINR is $29.48 d B$ on average which refers to a good signal level. Whereas, using the two other RPA schemes, the observed SINR is negative referring to a very bad signal quality. On the other hand, a thorough study of SINR level in Table IV confirms the aforementioned benefits of taking into consideration the macrocell communications. Despite the fact that the percentage of non-connected FUs of the TCRPA approach slightly exceeds those of QP-FCRA and DRAPM by nearly $0.5 \%$, almost all connections (i.e. $29.37 \%$ ) present a good SINR. On the other hand, QP-FCRA and DRPAM have only $1.3 \%$ of FUs with a good SINR $\geq 20 d B, 1.5 \%$ and $1.7 \%$ of FUs with a medium SINR level between $0 d B$ and $20 d B$, respectively, while the majority of connected users suffer of a negative SINR. The benefits of TCRPA are indeed confirmed by the results of final MUs' SINR in Table III where the approach preserves a very good signal quality to the coexisting macrocell network.
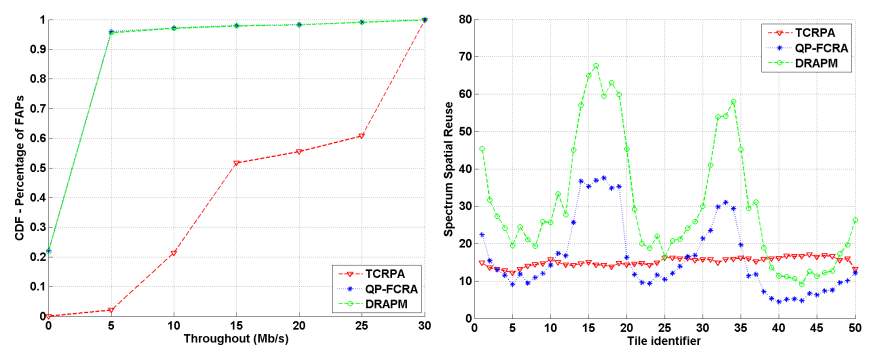

Fig. 1. Throughput per CDF of Fig. 2. Spectrum spatial reuse FAPs

2) Throughput analysis: Figure 1 reports the mean throughput per the cumulative distribution function of FAPs. We can observe that QP-FCRA and DRAPM present merely the same results. For both of them, about $96 \%$ of FAPs have a throughput less than $5 \mathrm{Mbps}$ while the percentage is only $3 \%$ for TCRPA. Moreover, only $4 \%$ of FAPs have between $5-30 M b p s$ as a throughput in the case of the comparison solutions. TCRPA allows about $50 \%$ of FAPs to have a throughout between $5-15 \mathrm{Mbps}$, $8 \%$ of FAPs to have a throughput between $15-25 \mathrm{Mbps}$, and $40 \%$ of them to have a throughput between $25-$ $30 \mathrm{Mbps}$. The important improvement of TCRPA in terms

\begin{tabular}{|l|l|l|}
\hline & $\begin{array}{l}\text { Mean FU } \\
\text { Throughput } \mathbf{( M b p s )}\end{array}$ & $\begin{array}{l}\text { Mean MU } \\
\text { Throughput (Mbps) }\end{array}$ \\
\hline TCRPA & $16.2336 \pm 0.0150$ & $45.3143 \pm 0.0223$ \\
\hline QP-FCRA & $0.7393 \pm 0.0099$ & $46.4399 \pm 0.0167$ \\
\hline DRAPM & $0.7777 \pm 0.0095$ & $44.7333 \pm 0.0092$ \\
\hline \multicolumn{2}{|c|}{ TABLE V }
\end{tabular}

THROUGHPUT MEAN VALUES

of throughput is undoubtedly confirmed by results reported in Table V. As shown, thanks to TCRPA, FUs can benefit of a throughput 20 times more important than the other approaches while keeping a similar comfortable throughput for MUs. This achievement is not surprising and is expected since the throughput is closely linked to the SINR as illustrated in Shanon Formula.

3) Spectrum spatial reuse analysis: It is obvious that one of the main objectives of a resource allocation scheme is to arise the re-usability of the available resources. As shown in Table VI, DRAPM outperforms the two semicentralized approaches by doubling its SSR compared to theirs. In fact, being a distributed approach, DRAPM let FAPs allocate to their FUs as much tiles as they want regardless their neighbors' needs. The selfishness of DRAPM explains its high SSR value. However, we demonstrated by the observations above that the more a tile is used while ignoring the interference impact, the more the value of the resource allocation approach is a fallacy.

\begin{tabular}{|l|l|l|}
\hline & Spectrum Spatial Reuse & Transmit power per tile (mW) \\
\hline TCRPA & $15.1244 \pm 0.0457$ & $1.0684 \pm 0.0026$ \\
\hline QP-FCRA & $16.0836 \pm 0.3865$ & $1.9311 \pm 0.0565$ \\
\hline DRAPM & $30.3166 \pm 0.6575$ & $3.6136 \pm 0.1118$ \\
\hline \multicolumn{3}{|c|}{ TABLE VI } \\
\hline \multicolumn{3}{|c|}{ SSR AND TRANSMIT POWER MEAN VALUES }
\end{tabular}

In spite of its relatively poor SSR compared to the two other approaches, Figure 2 reveals that TCRPA provides an interesting fairness between tiles. This can be explained by the cross-tiers interference that forbids FUs to transmit on the same tiles used by their potentially interfering MUs.

4) Power analysis: Studying the transmit power of FAPs is fundamental to judge the efficiency of our proposed joint power and resource allocation scheme. Hence, we dressed 3 plots in Figure 3. Figure 3 (a) represents the transmit power per tile. Observed results highlight how much fair is the TCRPA approach compared with the sawtooth-shaped curves of QP-FCRA and DRAPM schemes. Besides, Table VI reveals that the mean power per tile ensured by TCRPA can be less than a half and up to 3.6x less than the values obtained using QP-FCRA and DRAPM respectively. Using TCRPA, the mean transmit power of FAPs is around $1.06 \mathrm{~mW}$. This can be explained by the two conditions (a) and (d) of the MILP formulation. If the interference is important, a FAP has to transmit with a higher power. If we take into consideration both co-tier and cross-tier interference, power will be greater than if we only consider cotier interference. But, the power has a threshold that should not be surpassed. Thus, considering both types of interference may lead to a non transmission rather than a high-power-level one.

We next examine Figure 3 (b) that gives us an idea about the power value per the distance between the FU and its serving FAP. Logically, the power is in conjunction with the distance. So, an increase of the distance should induce an increase on the transmit power of 


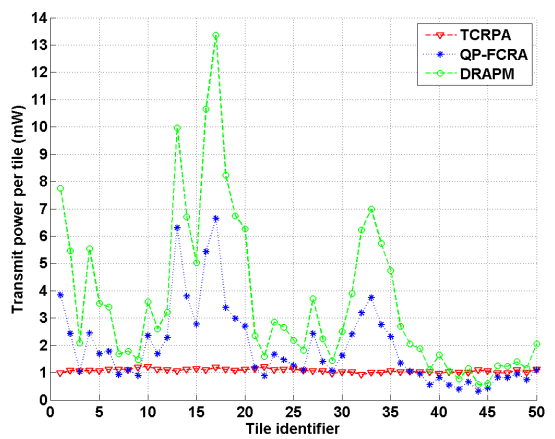

(a) Transmit power per tile identifier

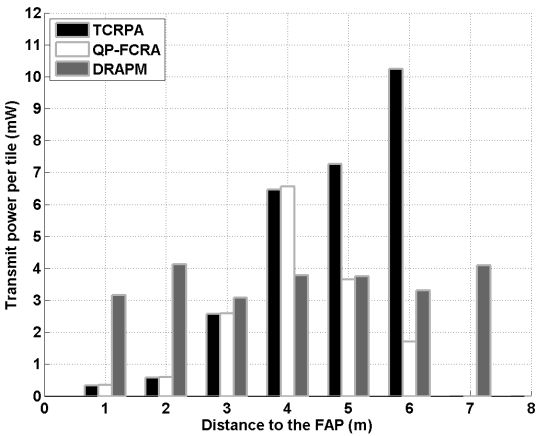

(b) Transmit power per distance

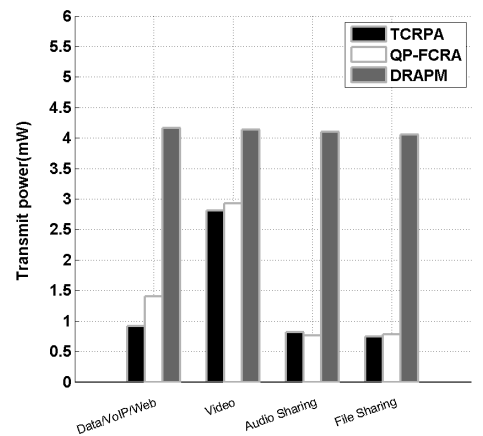

(c) Transmit power per application type

Fig. 3. Power study with SINR Threshold $=20 \mathrm{~dB}$

the FAP to effectively reach the FU that it serves.We notice that, for TCRPA and as it is supposed to be, the transmit power increases when the distance increases to rich $10 \mathrm{~mW}$ at $6 \mathrm{~m}$ which is the half of the maximum transmit power of the FAP. At $7 m$, FAPs apparently decide to not serve the FUs to avoid interference with a very high power that will be superior to $\frac{P_{\max }^{F}}{2}$. QP-FCRA gives almost the same results as our approach until $4 \mathrm{~m}$. Beyond this distance, which is the middle of the cell, the transmit power decreases. In fact, when the FUs are near to the cell edge where the cross-tier interference begins, they only consider the co-tier interference that starts to decrease. Whereas, using DRAP the power values are always around $3-4 m W$ regardless the distance. In fact, with this distributed approach, FAPs are not aware of the possible interfering transmissions of the macrocell and ignore a part of the co-layer possible interfering communications. Thus, the transmit power of a FAP will not be affected by the interference around.

Finally, Figure 3 (c) plots the effects of the application type on transmit power. It is apparent that DRAPM treats all applications alike even if they do not require the same bandwidth and delivers much power to FAPs than TCRPA and QP-FCRA. The two latter schemes clearly distinguish between high data rate and low data rate services. Though, TCRPA allows a less transmit power for Data/VoIP/Web and video which have more requirements in terms of bandwidth.

\section{CONCLUSION}

In this paper, we proposed a new resource and power allocation scheme for dense OFDMA-based femtocell networks called Two-tier Cluster-based Resource and Power Allocation (TCRPA). TCRPA is a cluser-based approach that takes into account the cluster members' QoS requirements and minimizes the transmit power for each of them, alleviating thus the interference between femtousers and macro-users. Simulation results show that our approach designed for femtocell networks outperforms the distributed solution DRAPM as well as QP-FCRA. The effectiveness of TCRPA was proved regarding to the final SINR level, the throughput, the spectrum spatial reuse, and the transmit power of FAPs. We also studied the performance of the macrocell layer to further confirm the gain of our approach. As next step, we plan to extend our work in order to allocate resources to macro-users and involve the users mobility.

\section{ACKNOWLEDGEMENT}

This work was partially supported by the ANR ABCD project (Grant no. ANR-13-INFR-0005-01) and the CNRS WINDS project (Grant no. 25995).

\section{REFERENCES}

[1] "Indoor deployment strategies," June 2014, NOKIA White Paper.

[2] N. Saquib et al., "Interference management in ofdma femtocell networks: issues and approaches," IEEE Wireless Communications, vol. 19, no. 3, pp. 86-95, June 2012.

[3] P. Palanisamy and S. Nirmala, "Downlink interference management in femtocell networks - a comprehensive study and survey," in International Conference on Information Communication and Embedded Systems (ICICES), Feb 2013, pp. 747-754.

[4] A. Mahmud and K. A. Hamdi, "Hybrid femtocell resource allocation strategy in fractional frequency reuse," in IEEE Wireless Communications and Networking Conference (WCNC), April 2013, pp. 2283-2288.

[5] M. Y. Arslan et al., "A resource management system for interference mitigation in enterprise ofdma femtocells," IEEE/ACM Transactions on Networking, vol. 21, no. 5, pp. 1447-1460, Oct 2013.

[6] A. Hatoum et al., "Qos-based power control and resource allocation in ofdma femtocell networks," in IEEE GLOBECOM 2012, Dec 2012, pp. 5116-5122.

[7] A. Abdelnasser et al., "Clustering and resource allocation for dense femtocells in a two-tier cellular ofdma network," IEEE Transactions on Wireless Communications, vol. 13, no. 3, pp. 16281641, March 2014.

[8] W. Li et al., "Dynamic clustering based sub-band allocation in dense femtocell environments," in Vehicular Technology Conference (VTC Spring), 2012 IEEE 75th, May 2012, pp. 1-5.

[9] Y. Zhang et al., "Clustering-based interference management in densely deployed femtocell networks," in IEEE/CIC International Conference on Communications in China (ICCC), Nov 2015, pp. 1-6.

[10] S. Jouili et al., "Median graph shift: A new clustering algorithm for graph domain," in International Conference on Pattern Recognition (ICPR), Aug 2010, pp. 950-953.

[11] A. Ladanyi et al., "Distributed resource allocation for femtocell interference coordination via power minimisation," in IEEE GLOBECOM Workshops (GC Wkshps), Dec 2011, pp. 744-749.

[12] "Cisco visual networking index: Global mobile data traffic forecast update, 2014-2019," 3 Feb 2016, CISCO White Paper.

[13] "Guidelines for evaluation of radio interface technologies for imtadvanced: Itu - r m.2135," 2008, Report. 